\title{
Non-characteristic Harmonic Analysis of Uhv ac/dc Systems
}

\author{
GONG Jie, ZHOU Lixing, ZHOU Lawu, ZHOU Mengdi \\ School of Electrical \& Information Engineering, Changsha University Of Science \& Technology, Changsha 410114, China
}

\begin{abstract}
Uhv direct current transmission has developed rapidly in China due to its unique advantages of large capacity, long distance and high voltage. A series of researches have been carried out on the non-characteristic harmonic current in the grid side of uhv dc transmission system. This paper is aimed at the analysis of noncharacteristic harmonics in the uhv direct current transmission system using a 12-pulse converter. Under ideal operating conditions, the system has characteristic non - characteristic harmonics. However, the actual operation condition of HVDC project is not ideal, so there are non-characteristic harmonics. The Simulink of MATLAB is used to model a uhv dc power transmission system. Based on this model, dynamic simulation is carried out to analyze the steady-state and dynamic characteristics of the system. Meanwhile, several common faults in the system are simulated. The simulation result is the same as the actual phenomenon, which provides support for identification, control and fault diagnosis of uhv ac/dc transmission system in the future.
\end{abstract}

\section{Before Uhv dc Transmission Converter Station}

With the development of national economy and the improvement of people's living standards, electric power and electronic products are widely used in the field of industrial control. These power electronic devices make the problem of harmonic pollution in power system more and more serious[1-3]. Therefore, the analysis and management of harmonic problem has become an important issue in the field of power system[4].

For uhv direct current transmission system, the important link is the commutation link and inverter link of the converter station[5-6]. Therefore, through a typical double-bridge rectifier station, the current characteristics of the uhv dc system were analyzed when operating under the working condition 4-5[7]. The both sides of the converter transformer $\mathrm{Y} / \mathrm{Y}$ type coupling current and $\mathrm{Y} / \Delta$ type connection of valve side current for $\mathrm{i}_{\mathrm{A} 1}$ (type 1 ), $\mathrm{Y} / \Delta$ net side current is group (2).

The series expansion formula of $i_{\mathrm{A} 1}$ is:

$$
\begin{aligned}
& i_{A 1}=\frac{2 \sqrt{3}}{\pi} i_{d}\left[\cos (w t)-\frac{1}{5} \cos (5 w t)+\frac{1}{7} \cos (7 w t)\right. \\
& -\frac{1}{11} \cos (11 w t)+\frac{1}{13} \cos (13 w t)-\frac{1}{17} \cos (17 w t) \\
& \left.+\frac{1}{19} \cos (19 w t)-\frac{1}{23} \cos (23 w t)+\frac{1}{25} \cos (25 w t) \cdots\right]
\end{aligned}
$$

The series expansion of $\mathrm{i}_{\mathrm{A} 2}$ is as follows:

$$
\begin{aligned}
& i_{A 2}=\frac{2 \sqrt{3}}{\pi} i_{d}\left[\cos (w t)+\frac{1}{5} \cos (5 w t)-\frac{1}{7} \cos (7 w t)-\frac{1}{11} \cos (11 w t)+\frac{1}{13} \cos (13 w t)\right. \\
& \left.+\frac{1}{17} \cos (17 w t)-\frac{1}{19} \cos (19 w t)-\frac{1}{23} \cos (23 w t)+\frac{1}{25} \cos (25 w t) \cdots\right]
\end{aligned}
$$

From equations 1 and 2, the expression of a-phase current is the sum of the above two terms: $\mathrm{i}_{\mathrm{A} 2}=2 \frac{2 \sqrt{3}}{\pi} i d\left[\cos (w t)-\frac{1}{11} \cos (11 w t)+\frac{1}{13} \cos (13 w t)-\frac{1}{23} \cos (23 w t)+\frac{1}{25} \cos (25 w t) \cdots\right]$

\section{Non-characteristic Harmonic Analysis of Uhv Direct Current Transmission}

This paper is aimed at the analysis of non-characteristic harmonics in the uhv direct current transmission system using a 12-pulse converter. Under ideal operating conditions, the system has characteristic non characteristic harmonics. But the actual operation condition of HVDC project is not possible [8-10].

The non-characteristic harmonic is defined as follows: "non-characteristic harmonic" is the component of the sine wave of a periodic electric quantity, whose frequency is integral multiple of the fundamental wave frequency. The sinusoidal voltage expression is as follows.

$$
u(t)=\sqrt{2} u \sin (w t+\varphi)
$$

Where, $\mathrm{u}$ is the effective value of voltage. $w t$ is the angular frequency, $\omega=2 \pi f=2 \pi / T, \varphi$ is the initial Angle. When the circuit is a non-sinusoidal circuit, a sinusoidal voltage is applied to the circuit. In general, the Fourier decomposition expansion of the non-sinusoidal voltage $\mathrm{u}(\mathrm{wt})$ of the period $\mathrm{T}=2 \pi / \omega$ is:

$$
u(w t)=a_{0}+\sum_{n=1}^{\infty}\left[a_{n} \cos (n w t)+b_{n} \cos (n w t)\right]
$$




$$
\begin{aligned}
& u(w t)=\sum_{n=1}^{\infty} c_{n} \sin \left(n w t+\varphi_{n}\right) \\
& a_{0}=\frac{1}{2 \pi} \int_{0}^{2 \pi} u(w t) d(w t) \\
& a_{n}=\frac{1}{\pi} \int_{0}^{2 \pi} u(w t) \cos (n w t) d(w t) \\
& b_{n}=\frac{1}{\pi} \int_{0}^{2 \pi} u(w t) \sin (n w t) d(w t) \\
& c_{n}=\sqrt{a_{n}^{2}+b_{n}^{2}} \\
& \varphi_{n}=\operatorname{arctg}\left(\frac{a_{n}}{b_{n}}\right) \\
& a_{n}=c_{n} \sin \varphi_{n} \\
& b_{n}=c_{n} \cos \varphi_{n}
\end{aligned}
$$

In the expression, the wave whose number is integer and larger than the fundamental wave is non-characteristic harmonic. For example, if the frequency of the fundamental wave is $50 \mathrm{~Hz}$ in China, then the second noncharacteristic harmonic is $100 \mathrm{hz}$, and the $\mathrm{n}$-th noncharacteristic harmonic is $50 \mathrm{nHz}$. If the fundamental wave is larger than the fundamental wave but not multiple of the fundamental wave, the wave between the fundamental wave frequency and the characteristic harmonic frequency is called inter-non-characteristic harmonic. The frequency lower than the fundamental frequency is called the sub-non - characteristic harmonics. The components of non-fundamental frequency multiples are called fractional non-characteristic harmonics. Voltage, frequency and waveform are the main indexes to measure the power quality, and they will seriously affect the operation of various electrical equipment and systems. Therefore, the power quality should be guaranteed in the normal operation system, so that the voltage, current and waveform can be restored to the sine wave. Therefore, the problem of non-characteristic harmonics and power quality in non-linear systems has a direct impact on the safe and economic operation of all kinds of power equipment. And in a power supply system, the voltage (or current) of the supply is always required to be sinusoidal.

\section{Common Methods for Non- Characteristic Harmonic Detection}

The instantaneous reactive power theory of three-phase circuit is proposed by Fryze, Quade and Akaji (Akaji et al.), which has been extensively and deeply studied and gradually improved. This theory breaks through the traditional definition of power based on average value, and systematically defines instantaneous power quantity such as instantaneous reactive power and instantaneous active power. Based on this theory, the real-time detection method of harmonic and reactive current for active power filter can be obtained.

The inspection method based on this theory plays a great role in the development of active power filter and is the most widely used method in APF. By calculating the instantaneous power of the load, including the dc component and the pulsating component, the pulsating part is separated by combining the historical data of a certain length, and the required reference signal is calculated according to the principle of average current in the three-phase.

The instantaneous reactive power theory of three-phase circuit was first proposed by chimu taiwen in 1983. $\alpha, \beta$ conversion: realize three phase instantaneous voltage, and the current is transformed from static to rotating. Orthogonal coordinate variables serve as the basis of analysis. Suppose the instantaneous values of voltage and current of each phase of three-phase circuit are $e_{a}, e_{b}, e_{c}$ and $i_{a}, i_{b}, i_{c}$ respectively. Let's apply them to the orthogonal coordinate system of $\alpha-\beta$.

$$
\begin{gathered}
{\left[\begin{array}{l}
e_{a} \\
e_{\beta}
\end{array}\right]=c_{32}\left[\begin{array}{l}
e_{a} \\
e_{b} \\
e_{c}
\end{array}\right]} \\
{\left[\begin{array}{l}
i_{a} \\
i_{\beta}
\end{array}\right]=c_{32}\left[\begin{array}{l}
i_{a} \\
i_{a} \\
i_{c}
\end{array}\right]}
\end{gathered}
$$

And

$$
c_{23}=\sqrt{\frac{2}{3}}\left[\left[\begin{array}{ccc}
1 & -1 / 2 & -1 / 2 \\
0 & \sqrt{3} / 2 & -\sqrt{3} / 2
\end{array}\right]\right]
$$

The $\alpha-\beta$ vector $e_{a}, e_{\beta}$ and $i_{a}, i_{\beta}$ shown in figure 1 can synthesize the voltage vector $e$ and current vector $i$, respectively:

$$
\begin{aligned}
& e=e_{a}+j \cdot e_{\beta}=|e| \angle \varphi_{e} \\
& i=i_{a}+j \cdot i_{\beta}=|i| \angle \varphi_{i}
\end{aligned}
$$

$|e|,|i|$ is the modulus of $e, i$ vector.

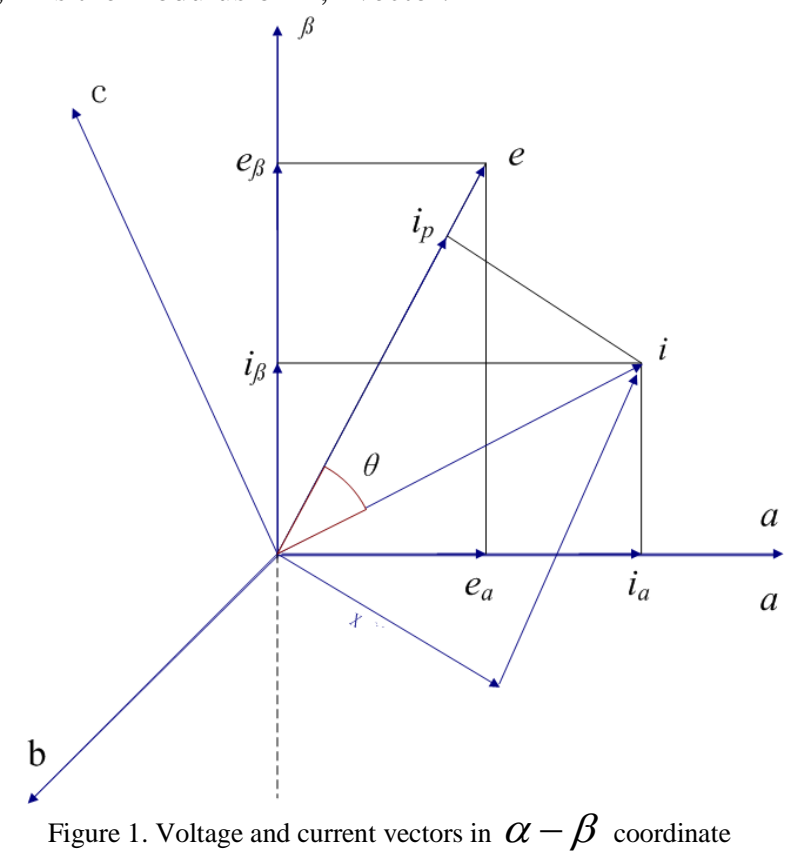
system

Three-phase instantaneous active power is:

$$
p=|e| \cdot|i| \cos \left(\varphi_{i}-\varphi_{e}\right)=|e| \cdot i_{p}=e \cdot i=e_{a} \cdot i_{a}+e_{\beta} i_{\beta}
$$


$q=|e||i| \sin \left(\varphi_{i}-\varphi_{e}\right)=|e| \cdot i_{q}=e \times i$

Three-phase instantaneous reactive power is:

$$
\left[\begin{array}{cc}
e_{\alpha} & e_{\beta} \\
i_{\alpha} & i_{\beta}
\end{array}\right]=e_{\alpha} i_{\beta}-e_{\beta} i_{\alpha}
$$

Three-phase instantaneous active power current is :

$$
i_{p}=|i| \cos \left(\varphi_{i}-\varphi_{e}\right)=\frac{e_{a} i_{a}+e_{\beta} i_{\beta}}{\sqrt{e_{a}^{2}+e_{\beta}^{2}}}
$$

The three-phase instantaneous reactive power current is :

$$
i_{q}=|i| \sin \left(\varphi_{i}-\varphi_{e}\right)=\frac{e_{a} i_{a}-e_{\beta} i_{\beta}}{\sqrt{e_{a}^{2}+e_{\beta}^{2}}}
$$

In matrix form

$$
\left[\begin{array}{l}
p \\
q
\end{array}\right]=\left[\begin{array}{cc}
e_{\alpha} & e_{\beta} \\
e_{\beta} & -e_{\alpha}
\end{array}\right]\left[\begin{array}{l}
i_{\alpha} \\
i_{\beta}
\end{array}\right]=c_{p q}\left[\begin{array}{l}
i_{\alpha} \\
i_{\beta}
\end{array}\right]
$$

$$
\left[\begin{array}{c}
i_{\alpha} \\
i_{\beta}
\end{array}\right]=\frac{1}{\sqrt{e_{\alpha}^{2}+e_{\beta}^{2}}}\left[\begin{array}{cc}
e_{\alpha} & e_{\beta} \\
e_{\beta} & -e_{\alpha}
\end{array}\right]\left[\begin{array}{c}
i_{\alpha} \\
i_{\beta}
\end{array}\right]=\frac{1}{\sqrt{e_{\alpha}^{2}+e_{\beta}^{2}}}\left[\begin{array}{l}
i_{\alpha} \\
i_{\beta}
\end{array}\right]
$$

$$
\text { And } \quad c_{p q}=\left[\begin{array}{cc}
e_{\alpha} & e_{\beta} \\
e_{\beta} & -e_{\alpha}
\end{array}\right]
$$

Now, it is assumed that the system three-phase voltage and current are all positive sequence fundamental wave sinusoids, and the three-phase voltage and current are set as:

$$
\begin{aligned}
& {\left[\begin{array}{l}
e_{a} \\
e_{b} \\
e_{c}
\end{array}\right]=\left[\begin{array}{l}
E_{m} \sin w t \\
E_{m} \sin (w t-2 \pi / 3) \\
E_{m} \sin (w t+2 \pi / 3)
\end{array}\right]} \\
& {\left[\begin{array}{l}
i_{a} \\
i_{a} \\
i_{c}
\end{array}\right]=\left[\begin{array}{l}
I_{m} \sin w t \\
I_{m} \sin (w t-\varphi-2 \pi / 3) \\
I_{m} \sin (w t-\varphi+2 \pi / 3)
\end{array}\right]}
\end{aligned}
$$

The corresponding vector in the $a-\beta$ coordinate system is:

$$
e=\left[\begin{array}{l}
e_{a} \\
e_{\beta}
\end{array}\right]=\sqrt{\frac{2}{3}}\left[\begin{array}{ccc}
1 & -1 / 2 & -1 / 2 \\
0 & \sqrt{3} / 2 & -\sqrt{3} / 2
\end{array}\right]\left[\begin{array}{c}
E_{m} \sin w t \\
E_{m} \sin (w t-2 \pi / 3) \\
E_{m} \sin (w t+2 \pi / 3)
\end{array}\right]=\sqrt{\frac{3}{2}} E_{m}\left[\begin{array}{c}
\sin w t \\
-\cos w t
\end{array}\right]
$$

$$
i=\left[\begin{array}{l}
i_{a} \\
i_{\beta}
\end{array}\right]=\sqrt{\frac{2}{3}}\left[\begin{array}{ccc}
1 & -1 / 2 & -1 / 2 \\
0 & \sqrt{3} / 2 & -\sqrt{3} / 2
\end{array}\right]\left[\begin{array}{c}
I_{m} \sin w t \\
I_{m} \sin (w t-2 \pi / 3) \\
I_{m} \sin (w t+2 \pi / 3)
\end{array}\right]=\sqrt{\frac{3}{2}} I_{m}\left[\begin{array}{c}
\sin (w t-\varphi) \\
-\cos (w t-\varphi)
\end{array}\right]
$$

Instantaneous active power is

$$
p=e \cdot i=\frac{3}{2} E_{m} I_{m} \cos \varphi
$$

The instantaneous reactive power is

$$
p=e \times i=\frac{3}{2} E_{m} I_{m} \sin (-\varphi)
$$

Let $E=E_{m} / 2, I_{m} / \sqrt{2}$ be the effective value of phase voltage and phase current respectively, then:

$$
p=3 E I \cos \varphi, q=3 E I \sin (-\varphi)
$$

It can be seen from the above that when the threephase voltage and current of the system are both positive sequence fundamental wave voltage and current, the instantaneous active power and reactive power calculated according to the above definition are consistent with the effective value calculation results of the usual three-phase active power and reactive power. The calculation of active and reactive power is defined on the basis of instantaneous value, so it is called instantaneous active power and instantaneous reactive power.

\section{$4 \quad$ Non-characteristic Suppression Methods}

Harmonic

Due to the existence of impedance in the system, when the ac side system of the converter station is injected with non-characteristic harmonics, the system will produce the voltage drop of each non-characteristic harmonics, which will cause the distortion voltage superposition with the sinusoidal wave, resulting in the generation of various system hazards and the adverse impact on various electrical equipment. The generation of non-characteristic harmonic voltage current in dc side affects the communication line around. In order to reduce the damage caused by non-characteristic harmonic voltage and current to the system and the environment, it needs to be suppressed by some methods.

There are two ideas on the basis of non-characteristic harmonic suppression: one is active suppression, that is, the device itself is modified from the non-characteristic harmonic source, so that the system no longer produces non-characteristic harmonic or reduces non-characteristic harmonic. Second, passive suppression, that is, the installation of additional devices, ultimately make the non-characteristic harmonics of the grid reduce or disappear. Among them, the method of increasing the phase number or the pulse number of the converter device is active. Passive filter PF and active filter APF are passive control measures.

Analysis before the characteristic harmonic frequency of the uhv dc transmission, for single bridge inverter pulse wave passing through Fourier analysis shows that the ac when increasing the pulse number of 12 pulse double bridge inverter, because it is a Yy joins six pulse converter transformer and a $\mathrm{Y}$ type delta connection combination of six pulse converter transformer, the network side of the converter transformer has 30 of phase three phase current. It can be clearly seen that the 5,7 non-characteristic harmonics produced by the 6 pulse converter are cancelled out. Based on this method, it can 
also be verified that 11 and 13 equal-order noncharacteristic harmonics in the 24-pulse converter can be offset, and the characteristic non-characteristic harmonics are only left with $24 \mathrm{k}$ times of non-characteristic harmonics. Therefore, the non-characteristic harmonic suppression can be achieved by increasing the pulse number of converter. The increase of pulse number can reduce the features of the harmonic characteristics of the components, so as to achieve the aim of inhibition of non characteristic harmonic, for example, in the chemical industry used in electrolytic six commonly used single converter transformer pulse and 12 pulse two kinds, the converter transformer stations more enterprises, Suggestions according to the pulse number of converter transformer, and the relationship between the phaseshifting Angle of 6 pulse and the combination of 12 pulse converter transformer properly, in order to effectively restrain the characteristic harmonic. 6 Pulse-changing rheological combination table and 12 Pulse-changing rheological combination table were shown in Table 1 and Table 2.

The phase can be formed when 6 or 12 pulsating converter transformers operate together in the manner given above the oscillating converter transformer operates to suppress non-characteristic harmonics.

At present, in the actual uhv direct current transmission engineering, 12 pulsations are mostly used in the converter, while 24 . This is because the structure of the corresponding converter composed of high pulsations is relatively complex, the equipment manufacturing is difficult, and the investment is relatively large. Compared with using filtering device to suppress non-characteristic harmonics, it cannot achieve economic effect.

\section{Analysis of Simulation Results}

Based on the uhv dc transmission model, the system steady-state, dc line fault and inverter side a ground fault are simulated respectively. The simulation results are as follows.

\section{Steady-state system waveform}

The simulated voltage and current waveform is shown in Figure 1. In the figure, $V_{D L}$ represents the dc line voltage, and $I_{D}$ and $I_{d r e f}$ represent the dc line current and the actual reference current respectively, both of which are the nominal values. Alpha is the trigger delay Angle of the rectifier. It can be seen that the system can run stably after a period of time. After steady state, the dc voltage is $1 \mathrm{pu}$ and the dc current is $1 \mathrm{pu}$.

Open DC side circuit breaker DC Fault module, set it to conduct at $0.7 \mathrm{~s}$ and disconnect at $0.75 \mathrm{~s}$, and the DC side grounding short circuit time is $0.05 \mathrm{~s}$. The correlation waveform of the rectifier side is shown in Figure 2, and the correlation waveform of the ac part is shown in Figure 3, Figure 4 analysis is as follows: when the dc side ground short circuit, the dc side current surges to about $2.5 \mathrm{pu}$, and the dc side voltage drops to 0 . By modulating the $\mathrm{dc}$ side protection module, the reference current dropped to $0.24 \mathrm{pu}$, so there is still dc current at the dc side after the fault occurs. At $t=0.772 \mathrm{~s}$, the trigger delay Angle is forced to be 1660 . The rectifier is running in the state of inverter, and the dc side voltage becomes negative, sending the energy of the circuit back to the ac system, causing the fault current to be quickly extinguished at zero. When $\mathrm{t}=0.822 \mathrm{~s}$, the trigger delay Angle is forced to cancel 1660. The dc side voltage and current returned to normal after $0.5 \mathrm{~s}$.

\section{Summary}

This paper first summarizes and prospects the methods of power harmonic detection and analysis at home and abroad, and then explains the basic concept and characteristic parameters of power harmonic. Additional additional losses from increased transmission, supply and use of electrical equipment; The influence on the scale meter; Influence on the work and reliability of relay protection and automatic device; The harm of harmonics on the use and life of household electrical equipment is discussed. The PSB of MATLAB and other toolkits (such as Simulink) can be used to model and simulate the dynamic characteristics of HVDC transmission system in the transient process conveniently and accurately, and the simulation time is short, which can display the changes of various parameters in real time and facilitate the intuitive analysis of system performance.

\section{Reference}

1. Gutierrez O, Fuerte-Esquivel C R, Rubio J A, et al. Harmonic analysis of AC/DC systems based on phase-domain multi-port network approach[J]. Electric Power Systems Research, 2008, 78(10):1789-1797.

2. Oliveira S E M D, Guimaraes J O R P. Effects of Voltage Supply Unbalance on AC Harmonic Current Components Produced by AC/DC Converters[J]. IEEE Transactions on Power Delivery, 2007, 22(4):2498-2507.

3. Liu B, Du Z, Li C. Harmonic power flow of VSCHVDC based AC/DC power systems[J]. Electric Power Systems Research, 2016, 133:355-364.

4. Mizuma T, Sagisaka Y, Neri K, et al. Harmonic analysis of synchronous machine with AC/DC converter, part 1. Harmonic voltage characteristics of AC/DC converter[J]. Electrical Engineering in Japan, 2010, 105(3):71-79.

5. Vittek J, Najjar M Y. Common methodology for steady state harmonic analysis of inverters[J]. IEEE Transactions on Power Delivery, 2002, 10(3):16281634.

6. Xie G L, Zhang B H, Li Y, et al. Harmonic Propagation and Interaction Evaluation between Small-Scale Wind Farms and Nonlinear Loads[J]. Energies, 2013, 6(7):3297-3322.

7. Moya A A. Harmonic analysis in ideal ion-exchange membrane systems[J]. Electrochimica Acta, 2013, 90(5):1-11.

8. Drozhdin S N, Kamysheva L N, Kuyantseva O N. Investigations of the dynamical unipolarity of DTGS crystals by means of harmonic analysis of the switching current $[J]$. Ferroelectrics, 2000, 238(1):3339. 
9. Varricchio S L, Jr S G, Rangel R D. Three winding transformer -domain model for modal analysis of electrical networks[J]. International Journal of Electrical Power \& Energy Systems, 2011, 33(3):420-429.

10. Wang $M$ H, Huang $M$ L, Jiang W J. Maximum Power Point Tracking and Harmonic Reducing
Control Method for Generator-Based Exercise Equipment[J]. Energies, 2016, 9(2):103

Table 1. 6. Pulse-changing rheological combination table

\begin{tabular}{|c|c|c|c|c|c|c|}
\hline $\begin{array}{c}\text { Number of converter } \\
\text { transformer }\end{array}$ & 2 & 3 & 4 & 5 & 6 & 8 \\
\hline Phase-shifting Angle & $30^{\circ}$ & $20^{\circ}$ & $15^{\circ}$ & $12^{\circ}$ & $10^{\circ}$ & $7.5^{\circ}$ \\
\hline $\begin{array}{c}\text { Minimum feature non - } \\
\text { feature harmonic frequency }\end{array}$ & 11,13 & 17,19 & 23,25 & 29,31 & 35,37 & 47,49 \\
\hline
\end{tabular}

Table 2. 12 Pulse-changing rheological combination table

\begin{tabular}{|c|c|c|c|}
\hline Number of converter transformer & 2 & 3 & 4 \\
\hline Phase-shifting Angle & $15^{\circ}$ & $10^{\circ}$ & $7.5^{\circ}$ \\
\hline Minimum feature non - feature harmonic frequency & 23,25 & 35,37 & 47,49 \\
\hline
\end{tabular}

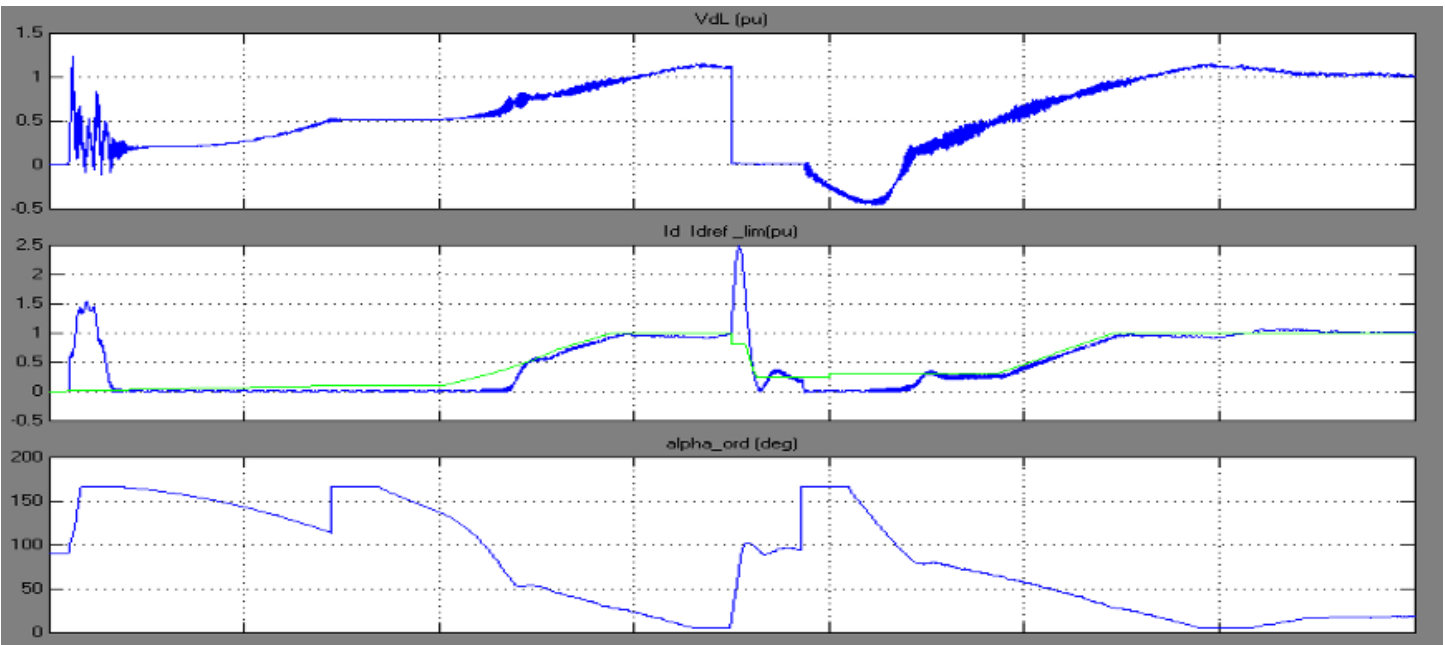

Figure 2. Rectifying side correlation waveformw 


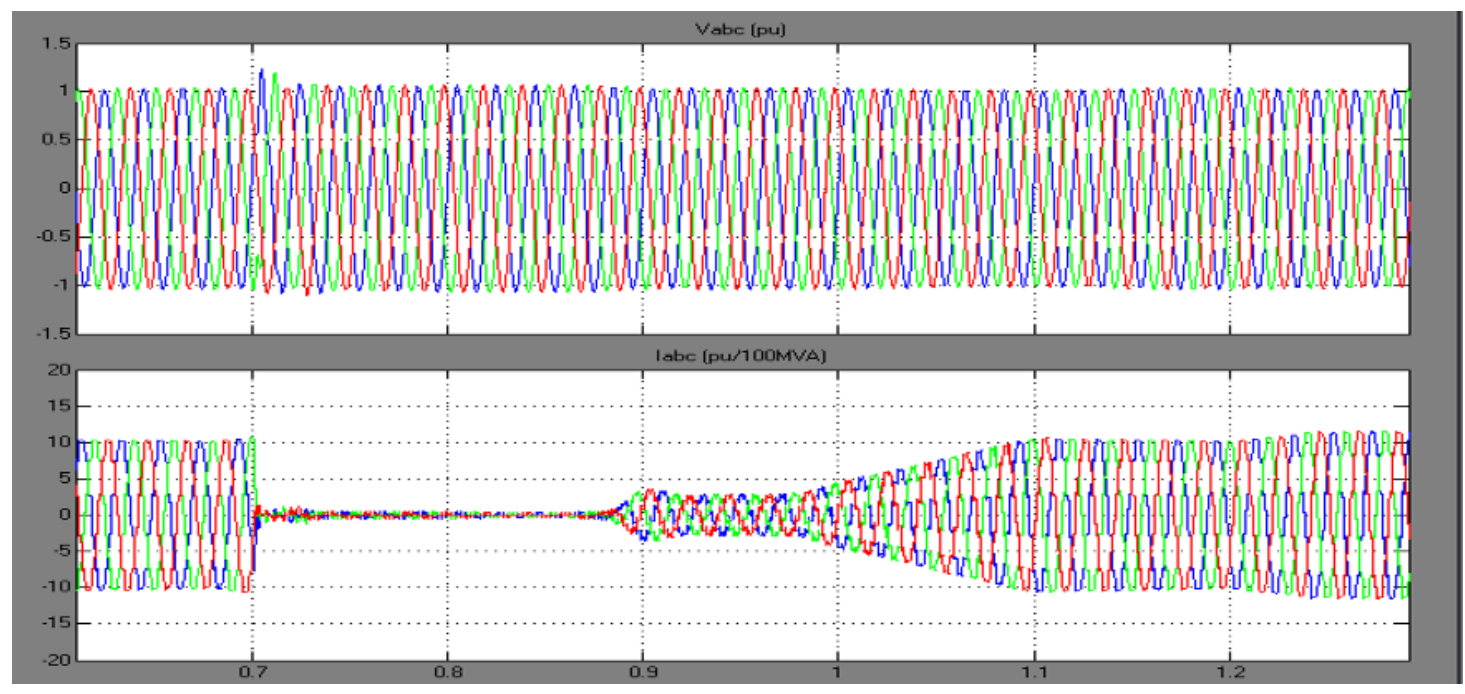

Figure 3. Correlation waveform of ac section

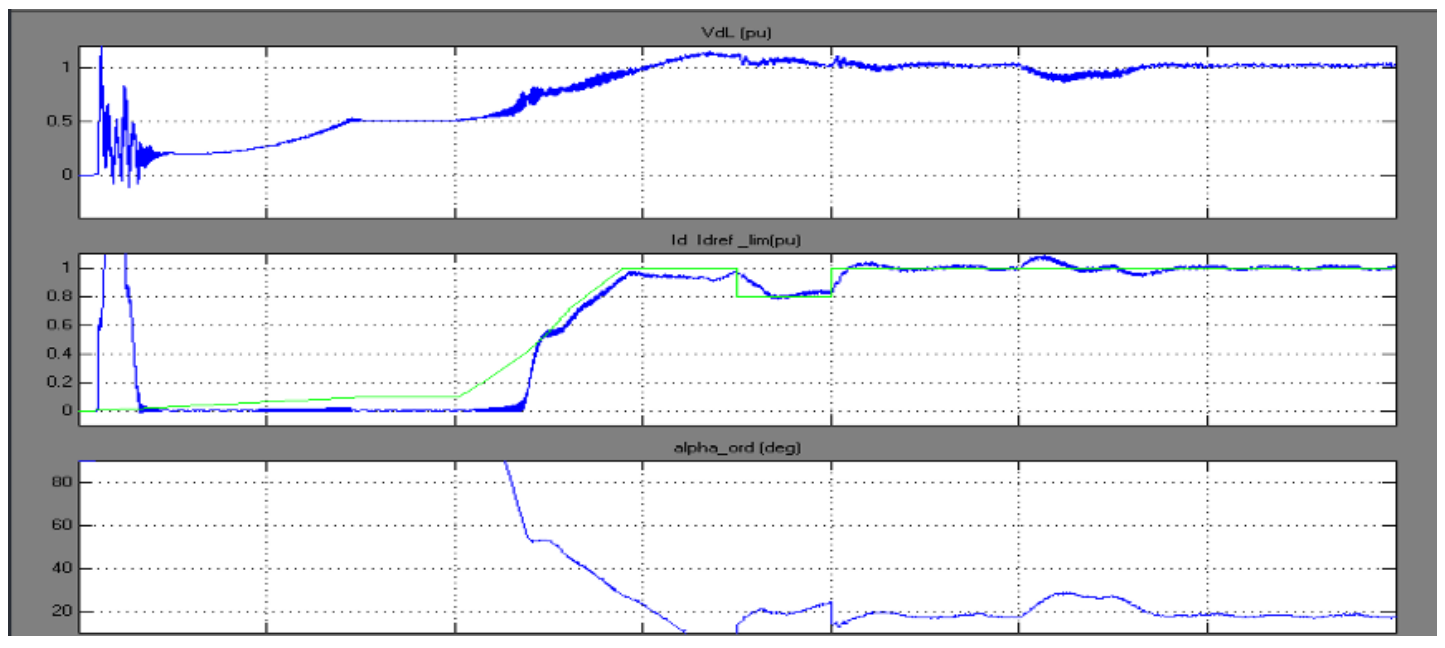

Figure 4. De side waveform of steady-state system $5.2 \mathrm{dc}$ line fault 